\title{
A High-Dynamic-Range Integrated Continuous-Time Bandpass Filter
}

\author{
Gert Groenewold
}

\begin{abstract}
An eighth-order Butterworth bandpass filter, operating at $100 \mathrm{kHz}$ with a quality factor of 14.3 , is presented. The filter features an optimized dynamic range, a large tuning range, and a small occupied chip area of $0.25 \mathrm{~mm}^{2}$ owing to very simple circuitry. Measurements show a very accurate realization of the desired transfer function, a high dynamic range of $62 \mathrm{~dB}$, and a tuning range from 50 to $200 \mathrm{kHz}$. It is shown how the dynamic range can be improved to a theoretical maximum if circuit simplicity is sacrificed.
\end{abstract}

\section{INTRODUCTION}

$\mathrm{A}$ $\mathrm{N} n^{\text {th }}$-order integrated analog continuous-time filter consists of a network of $n$ integrators. Therefore, the design process of the filter comprises the design of the integrator and of the network configuration. Several difficulties have to be coped with. One of these is the inaccuracy of component values. One overcomes this problem by making the filter electronically tunable and furnishing it with an automatic tuning circuit [1]. The filter is made tunable by making the integrator tunable.

This introduces another problem because in practice one sees that tunable integrators are nonlinear. This nonlinearity gives rise to distortion and thus limits the maximal signal level the integrator can handle. This, in turn, limits the dynamic range-which is defined as the ratio of the maximal signal level to the minimal signal level that the circuit in question can handle-of the integrator, and thus of the filter. The minimal signal level is determined by the noise of the circuit, the maximal signal level by distortion.

To phrase it more exactly, distortion limits the distortion-free dynamic range of a circuit. The maximal signal level in the distortion-free dynamic range is the signal level where the level of the distortion products is equal to the noise level. If the maximal signal level is determined by clipping effects, one talks about dynamic range. In both definitions the minimal signal level is the noise level. The distortion-free dynamic range can never be larger than the dynamic range, and usually is some tens of decibels smaller.

In many applications it is very difficult or even impossible to obtain a dynamic range or distortion-free dynamic

Manuscript received November 26, 1991; revised June 12, 1992. This work was supported by the Dutch IOP fund.

The author is with the Department of Electrical Engineering, Electronics Research Laboratory, Delft University of Technology, 2628 CD Delft, The Netherlands.

IEEE Log Number 9203553. range that is large enough. Especially in the case of bandpass filters with a high quality factor $Q$, which is defined as the ratio of the central frequency to the bandwidth of the filter, (distortion-free) dynamic range is a problem, because it can be proven [2] that the dynamic range of a filter of this class is inversely proportional to its quality factor. Therefore in some applications one should do everything possible to optimize the dynamic range of bandpass filters.

It is not difficult to show [3], [4] that the larger the supply voltages and the larger the capacitances that are available, the larger the dynamic range that can be obtained. In Table I an overview is given of the performance of MOSFET-based low-pass filters, as reported in literature. An attainable fundamental maximum for the dynamic range of the filters has been calculated from their respective transfer functions, total capacitance values, and supply voltages. These values are tabulated as $D R_{\text {opt }}$. The difference between the maximal and the actual dynamic range is specified as $D R_{\mathrm{dif}}$. The latter value is a figure of merit for the filter realization in question. Comparing the filter realizations in Table I on the basis of this figure of merit, we see that op-amp filters are better than transconductance filters. In practice, op-amp filters can be made to have a larger dynamic range than transconductance filters, but from a strictly theoretical point of view this is not true [3], [4].

For (MOSFET-based) bandpass filters, similar results are summarized in Table II. We see that the dynamic range of bandpass filters is lower than the dynamic range of lowpass filters, which could be expected from the $Q$ dependence of the dynamic range mentioned above.

It must be noted that the values for the dynamic range of the filters in Tables I and II have in some cases been recalculated to make a fair comparison possible. Dynamic-range reductions by test-signal feedthrough have in all cases been discarded, and the output noise has been determined in a broad band (theoretically from $-\infty$ to $\infty)$.

In this article we present an eighth-order Butterworth bandpass filter that has been realized in BiCMOS technology. This filter was intended partly for the realization of on-chip selectivity for radios, and partly for the demonstration of the viability of new design theories for integrated continuous-time filters with an optimal dynamic range [2]-[4]. Its central frequency (in radians per second) is nominally $2 \pi \cdot 100 \mathrm{kHz}$, its quality factor is 14.3 , 
TABLE I

an Overview of Dynamic-Range Performances of MOSFet low-Pass Filters from literature. In the Second Column, $T$ Stands for Transconductance-Based Filters, and $O$ for Op-Amp-MOSFET-C Based Filters. $D R$ is the Dynamic Range; SPECIFIEd are the Dynamic Range of the Filter, a Fundamental Optimum for the Dynamic Range, and the Difference Between These Two.

\begin{tabular}{|c|c|c|c|c|c|c|c|c|c|c|}
\hline \multirow[b]{2}{*}{ Reference } & \multirow[b]{2}{*}{$\mathrm{T} / \mathrm{O}$} & \multirow[b]{2}{*}{ Order } & \multirow{2}{*}{$\begin{array}{l}\text { Supply } \\
\text { Voltage } \\
\text { (V) }\end{array}$} & \multirow{2}{*}{$\begin{array}{c}\text { Total } \\
\text { Capacitance } \\
\text { (pF) }\end{array}$} & \multicolumn{2}{|c|}{$\begin{array}{c}\text { Area } \\
\left(\mathrm{mm}^{2}\right)\end{array}$} & \multicolumn{3}{|c|}{$\begin{array}{c}\text { DR } \\
\text { (dB) }\end{array}$} & \multirow[b]{2}{*}{ Year } \\
\hline & & & & & chip & filter & filter & opt & dif & \\
\hline [5] & $\mathbf{T}$ & 5 & 24 & approx. 100 & 6.5 & & 85 & 108 & 23 & 1978 \\
\hline [6] & $\mathrm{O}$ & 5 & 10 & 197 & 4 & & 95 & 104 & 9 & 1983 \\
\hline [7] & 0 & 5 & 10 & 358 & 4 & & 100 & 106 & 6 & 1985 \\
\hline$[8]$ & $\mathrm{T}$ & 3 & 5 & 28 & 1 & 0.66 & 70 & 93 & 23 & 1988 \\
\hline [9] & $T$ & 3 & 3 & 72 & 1 & & 63 & 94 & 31 & 1989 \\
\hline$[10],[11]$ & $\mathbf{T}$ & 3 & 8 & 14.5 & & 0.63 & 70 & 94 & 24 & 1990 \\
\hline [12] & $\mathbf{T}$ & 7 & 5 & $?$ & 6 & & 61 & $?$ & $?$ & 1990 \\
\hline [13] & $\mathrm{O}$ & 5 & 8 & 1078 & 3.6 & 2.5 & 98 & 110 & 12 & 1991 \\
\hline [14] & $\mathrm{O}$ & 5 & 5 & 73.5 & & 0.3 & 70 & 93 & 23 & 1991 \\
\hline [15] & $\mathrm{T}$ & 4 & 5 & 88.5 & & 0.92 & 66 & 95 & 29 & 1991 \\
\hline [16] & $\mathrm{T}$ & 2 & 5 & 4.6 & & 0.32 & 75 & 86 & 11 & 1991 \\
\hline [17] & $\mathrm{O}$ & 4 & 10 & approx. 700 & 43 & & 95 & 112 & 17 & 1991 \\
\hline
\end{tabular}

TABLE II

An Overview of Dynamic-Range Performances of MOSfet Bandpass Filters from Literature

\begin{tabular}{|c|c|c|c|c|c|c|c|c|c|c|c|}
\hline \multirow[b]{2}{*}{ Reference } & \multirow[b]{2}{*}{$\mathrm{T} / \mathrm{O}$} & \multirow[b]{2}{*}{ Order } & \multirow{2}{*}{$\begin{array}{l}\text { Supply } \\
\text { Voltage } \\
\text { (V) }\end{array}$} & \multirow{2}{*}{$\begin{array}{c}\text { Total } \\
\text { Capacitance } \\
(p F)\end{array}$} & \multirow[b]{2}{*}{$Q$} & \multicolumn{2}{|c|}{$\begin{array}{c}\text { Area } \\
\left(\mathrm{mm}^{2}\right)\end{array}$} & \multicolumn{3}{|c|}{$\begin{array}{c}\text { DR } \\
(\mathrm{dB})\end{array}$} & \multirow[b]{2}{*}{ Year } \\
\hline & & & & & & chip & filter & filter & opt & dif & \\
\hline [18] & $\mathrm{T}$ & 6 & 10 & 288 & 5.2 & 4 & & 70 & 96 & 26 & 1984 \\
\hline [19] & $\mathrm{T}$ & 8 & 10 & approx. 60 & 5 & 23 & & 45 & 86 & 41 & 1988 \\
\hline$[20]$ & $\mathrm{T}$ & 2 & 10 & $?$ & 10 & 0.3 & 0.09 & 56 & $?$ & $?$ & 1988 \\
\hline This Work & $\mathrm{T}$ & 8 & 8 & 80 & 14.3 & 1.1 & 0.25 & 62 & 83 & 21 & 1992 \\
\hline
\end{tabular}

TABLE III

The Design OBjectives

\begin{tabular}{cc}
\hline Supply Voltage & \\
Order & $8 \mathrm{~V}$ \\
Type & 8 \\
Total Capacitance & Butterworth \\
Central Frequency & $80 \mathrm{pF}$ \\
Bandwidth & $2 \pi \cdot 100 \mathrm{kHz}$ \\
$Q$ & $2 \pi \cdot 7 \mathrm{kHz}$ \\
\hline
\end{tabular}

so its nominal bandwidth is $2 \pi \cdot 7 \mathrm{kHz}$. Table III shows the design objectives of the filter. An additional design objective was to realize the filter with very simple circuits that occupy a small amount of chip area, but retain a high dynamic range. We chose for a transconductor approach because transconductors are simpler and occupy less chip area than op amps. A drawback of transconductors is that in practice they result in a smaller dynamic range than op amps can do. This is compensated by the fact that the filter network has been optimized with respect to dynamic range. This resulted in an eighth-order filter with a dynamic range of $62 \mathrm{~dB}$, occupying a chip area of only 0.25 $\mathrm{mm}^{2}$. In short, the features of the filter are:

- simple circuitry,

- a small chip area,

- a large tuning range,

- a high dynamic range,

- an accurate realization of the transfer function.
We proceed with discussing the tunable integrator circuit that was applied. Then, after some remarks on the use of dynamic range optimal filter networks, an explanation is given of the design of the filter, using the integrator as a building block in a network that is close to the optimum. Measurements are discussed, and it is shown what the maximal dynamic range is of this filter, if not only the filter network has been optimized, but also the integrators. Conclusions follow.

\section{The InTEgrator}

Fig. 1 shows the integrator we used. It consists of a transconductance stage and a capacitor. The kernel of the transconductor is transistor $T_{1}$, which is biased in the triode region. The drain voltage of this transistor is kept to a constant value via $T_{2}$. According to a simple model that relates the drain current $I_{d}$ of $T_{1}$ to its gate-to-source voltage $V_{g s}$ and its drain-to-source voltage $V_{d s}[21]$ :

$$
I_{d}=\frac{W}{L} \mu C_{o x}^{\prime}\left(\left(V_{g s}-V_{t}\right) V_{d s}-\frac{1}{2}(1+\delta) V_{d s}^{2}\right) .
$$

Here $W$ and $L$ are the width and length of the transistor, respectively, $\mu$ is the electron mobility, $C_{o x}^{\prime}$ is the oxide capacitance per unit area, $V_{t}$ is the threshold voltage, and $\delta$ is the bias-dependent parameter with a value of about 0.12 in this context. For the 2.5- $\mu \mathrm{m}$ Philips BiCMOS process that was used, the transconductance factor $\mu C_{o x}^{\prime}$ is 


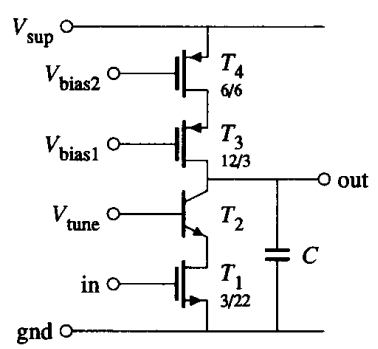

Fig. 1. The integrator, which consists of a transconductance stage and a capacitor.

$45 \mu \mathrm{A} / \mathrm{V}$ for n-channel MOSFET's, and $15 \mu \mathrm{A} / \mathrm{V}$ for p-channel MOSFET's. To designate a parameter of a specific transistor, we will add the index number of this transistor as an extra subscript, as in $I_{d_{1}}$ for $I_{d}$ of $T_{1}$.

From (1) we see that if $V_{d s}$ is constant, $I_{d}$ has a linear relationship with $V_{g s}$. The nonlinearity of this integrator comes into view if a more sophisticated model than the one employed above is used. It then appears that the mechanism referred to as "mobility reduction" is responsible for the nonlinearity and distortion [22] The transconductance can be tuned via $V_{d s}$. This principle of using a gate-driven MOSFET operated in the triode region has been initiated by Pennock [23], and has also been used by Wong [24], Steyaert et al. [15], and Alini et al. [16].

For maximizing the dynamic range of this stage, its noise production should be minimized, and its signal-handling capacity should be maximized. The signal-handling capacity is dependent on the bias voltages of the transistors. That is, the maximal peak-to-peak output signal voltage is equal to the supply voltage minus the drainsource voltage of $T_{1}$ minus the sum of the saturation drainsource voltages of $T_{2}$ to $T_{4}$. When these bias voltages are increased, the maximum output signal level decreases ultimately to zero, so that the dynamic range also decreases to zero.

The (double-sided) input-referred noise voltage spectrum of the integrator is

$$
S_{n, i}=2 k T \xi / G
$$

in which $k$ is Boltzmann's constant, $T$ is the absolute temperature, and $G$ is the transconductance of the stage. This equation implicitly defines the noise factor $\xi$. The definition has been chosen such that the noise factor of a passive conductor $G$ is 1 , so that in practice the noise factor of an integrator can never be made smaller than 1 . An integrator with a fundamentally optimal dynamic range therefore has $\xi=1$. The dynamic range of this integrator and of the filter are inversely proportional to $\xi$.

The noisy components in Fig. 1 are transistors $T_{1}$ and $T_{4}$. A simple expression for the input-referred noise voltage of these transistors is [21]

$$
\begin{aligned}
S_{n i}^{\prime}(\omega)= & 2 k T\left(c \frac{(1+\delta)}{(W / L) \mu C_{o x}^{\prime}\left(V_{g s}-V_{t}\right)}\right. \\
& \left.\cdot \frac{1+\alpha+\alpha^{2}}{\left(1-\alpha^{2}\right)(1-\alpha)}\right)
\end{aligned}
$$

in which

$$
\alpha=1-\frac{(1+\delta) V_{d s}}{V_{g s}-V_{t}}
$$

in the triode region, and $\alpha=0$ in the saturation region. In theory, $c$ has a value of $2 / 3$, but in practice larger values are found. The noise production of $T_{1}$ (with constant $G$ ) decreases if its drain-source voltage is increased. $T_{4}$ supplies a bias current accompanied by a noise current. This noise current can be decreased if the drain-source voltage of this transistor is increased. In this way it can be seen that the noise factor and the dynamic range decrease to zero if the drain-source voltages of $T_{1}$ and $T_{4}$ are decreased to zero.

Apparently, for a maximal dynamic range, the drainsource voltages of $T_{1}$ and $T_{4}$ must be neither very large nor very small, and optimal values can be found [3], [4]. The saturation voltage of $T_{3}$ (approximated by $\left(V_{g s}-V_{r}\right)_{3}$ ) must be as small as possible to leave maximal headroom for $T_{1}$ and $T_{4}$; a practical minimum before weak-inversion effects occur is $0.5 \mathrm{~V}$. Further optimization yielded $1.3 \mathrm{~V}$ for $V_{d s_{1}}, 1.6 \mathrm{~V}$ for $\left(V_{g s}-V_{t}\right)_{4}$, and $4 \mathrm{~V}$ for $V_{g s_{1}}$, if the supply voltage $V_{\text {sup }}$ is $8 \mathrm{~V}$. With these values, (2), and (3), assuming $c=2 / 3, \xi$ is found to be 5.2 . (Because in some cases an extra invertor must be added to realize a noninverting transfer, the effective noise factor increases to 6.6. This is further explained in Section IV.) The bias current is $15 \mu \mathrm{A}$.

The bias voltages required by the integrator are generated by the circuit in Fig. 2 . The values for $V_{\text {sup }}, V_{\text {tune, }}$, and $V_{\text {bias }}$ are nominally $8,1.9$, and $4 \mathrm{~V}$, respectively. $T_{101}$ in the bias circuit matches $T_{1}$ in the integrator, and $T_{104}$ matches $T_{4}$. In a quiescent situation, the current through the capacitor $C$ should be zero, so the drain currents of $T_{1}$ and $T_{4}$ should have equal magnitude. Because the drain currents of $T_{101}$ and $T_{104}$ are equal in magnitude, this is achieved if the drain currents of $T_{101}$ and $T_{104}$ match those of $T_{1}$ and $T_{4}$, respectively. For $T_{1}$ this means that its terminal voltages must be the same as the terminal voltages of $T_{101}$, so that, in a quiescent situation, the gate voltage of $T_{1}$ equals $V_{\text {bias }}$. This gate voltage is set via the internal feedback in any filter that is built up with these integrators. Matching of the drain currents of $T_{4}$ and $T_{104}$ is achieved by biasing these transistors with the same gatesource voltage. As these transistors are biased in the saturation region, their drain-source voltages do not need to be the same. The difference between $V_{\text {bias1 }}$ and $V_{\text {bias2 }}$ is set by the junction voltage $V_{b e}$ of $T_{103}$. If this junction voltage is larger than (approximately) $V_{g s 3}-V_{t 4}, T_{4}$ is in saturation. Using a junction voltage of a bipolar transistor for this purpose is not conventional. Usually, a diode-connected PMOS transistor is used for this purpose. If this PMOS transistor matches $T_{3}$, the drain-source voltage of $T_{4}$ would match the drain-source voltage of $T_{104}$. As it is arranged here, the drain-source voltage of $T_{4}$ is lower in magnitude. This has the advantage that the maximal signal level at the output of the integrator is larger, so that the dynamic range is larger. 


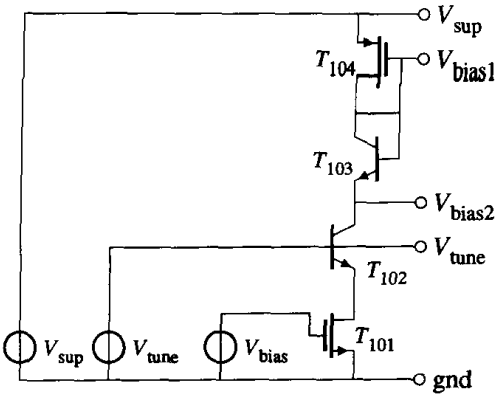

Fig. 2. The bias circuit.

The distortion-free dynamic range depends on the noise and distortion characteristics of the circuit. To guarantee distortion-free operation according to (1), the drain voltage of $T_{1}$ must be constant. The distortion properties of this stage depend on the measure in which this demand is met. As (1) has limited accuracy, measurements (published in [4] and to be published in [22]) show that even if this demand is met, distortion arises. These measurements match distortion measurements on this stage, so that we may conclude that the effects of spurious signal-induced voltage fluctuations on the drain of $T_{1}$ are negligible.

The integrator has little distortion, and the distortionfree dynamic range was measured to be as high as $74 \mathrm{~dB}$.

The maximum input amplitude before clipping is $1 V_{\text {eff }}$ This makes the dynamic range $85 \mathrm{~dB}$.

\section{The Filter Network}

In the previous section the design of the integrator was described. This integrator is to be used in a filter network. The design of a filter network is discussed below.

For the realization of any transfer function, many filter networks are possible. The dynamic range of the filter is dependent on the filter network that is chosen. It is possible to design an optimal network that can be proven to give rise to an optimal dynamic range. These optimal networks are quite difficult to realize, due to the many branches that are involved. Therefore, we use a simpler network that can be proved to come very close to this optimum. The theory on optimal dynamic range networks has been published elsewhere [2]; we suffice here by mentioning the main results.

A bandpass filter can be designed by performing a lowpass to bandpass transformation on a so-called low-pass equivalent filter. We start by designing a low-pass filter of the preferred type (Butterworth), with an order that is half the order of the bandpass filter we want to end up with. We choose a low-pass equivalent filter that is normalized, which means that it has a dimensionless bandwidth of 1 . Let $s$ " be the (dimensionless) Laplace operator for the normalized low-pass equivalent case, and $s$ the Laplace operator for the bandpass case (with the dimension of a frequency). Then the transformation that trans- forms this filter into the desired bandpass filter is

$$
s^{\prime \prime}=\frac{s^{2}+\omega_{0}^{2}}{s \omega_{c}}
$$

where $\omega_{0}$ is the central frequency of the bandpass filter, and $\omega_{c}$ is its bandwidth. The quality factor of the resulting filter is then

$$
Q=\frac{\omega_{0}}{\omega_{c}} .
$$

It can be proved that if we start by designing a low-pass equivalent filter that is close to optimum, after low-pass to bandpass transformation we end up with a bandpass filter that is just as close to optimum [2]. An optimal network for the low-pass equivalent filter can be found by starting with some network that realizes the transfer function that is wanted, and performing network optimization steps afterwards.

Network optimization can be done in two ways: scaling and network transformation. Scaling is a network operation that preserves the graph of the network, but it changes the branch values of the graph such that all the signal levels within the graph become equal. A network transformation goes further and also changes the graph. In fact, network transformations include scaling operations. They can give rise to a graph that is badly realizable, due to its complexity. Therefore it is wise to start with a graph that is known to "behave well," that is, it results in a nearoptimal network after scaling so that further network transformations are unnecessary.

The signal flow graph of the network that we have chosen for the low-pass equivalent filter is shown in Fig. 3. The network consists of a cascade of two biquads. Each biquad contains two integrators, which are presented in the figure by the branches that are marked with $1 / s^{\prime \prime}$.

One can prove [2] that, if this network is used, the dynamic range of the resulting bandpass filter after scaling is

$$
D R=0.0638 \frac{V_{\max , \mathrm{eff}}^{2} C}{4 k T \xi Q}
$$

where $V_{\text {max, eff }}$ is the maximal effective (root mean square) value of the single-ended signals at the inputs or at the outputs of the integrators that are used in the realization. $C$ is the total capacitance that is used in the filter. It can also be proved that the dynamic range belonging to an optimal network is

$$
D R_{\text {opt }}=0.0735 \frac{V_{\text {max, } \mathrm{eff}}^{2} C}{4 k T \xi Q} .
$$

This is only $0.6 \mathrm{~dB}$ above the dynamic range that is realized via the scaled cascade network, so that this network is very suitable. By (7), with $T=300 \mathrm{~K}, C=80 \mathrm{pF}$, $V_{\max , \text { eff }}=1 \mathrm{~V}, \xi=6.6$, and $Q=14.3$, the dynamic range of the bandpass filter is $65.1 \mathrm{~dB}$. The branch values of the scaled network are given in Table IV.

The effect of the frequency transformation (5) is that 


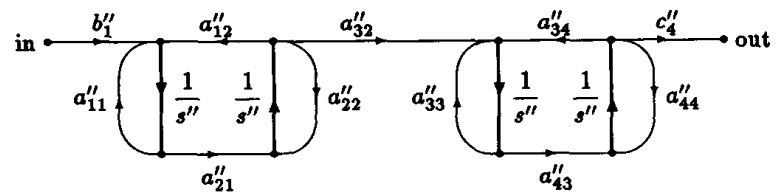

Fig. 3. The low-pass equivalent filter network: a cascade. The double primes are meant to indicate that this is a normalized low-pass equivalent.

TABLE IV

The Branch Values of the Network of Fig. 3 after Scaling

\begin{tabular}{lr}
\hline branch & \multicolumn{1}{c}{ value } \\
\hline$a_{11}^{\prime \prime}$ & -0.3827 \\
$a_{12}^{\prime \prime}$ & -0.7972 \\
$a_{21}^{\prime \prime}$ & 1.0708 \\
$a_{22}^{\prime \prime}$ & -0.3827 \\
$a_{32}^{\prime \prime}$ & 1.2560 \\
$a_{33}^{\prime \prime}$ & -0.9239 \\
$a_{34}^{\prime \prime}$ & -0.1301 \\
$a_{43}^{\prime \prime}$ & 1.1260 \\
$a_{44}^{\prime \prime}$ & -0.9239 \\
$b_{1}^{\prime \prime}$ & 1.1555 \\
$c_{4}^{\prime \prime}$ & 1.0000 \\
\hline
\end{tabular}
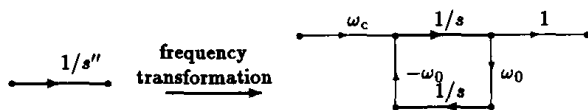

Fig. 4. The frequency transformation (5) can be realized by replacing each integrator of the normalized low-pass equivalent filter by a biquad.

each integrator branch with transfer function $1 / s^{\prime \prime}$ in the graph of Fig. 3 is replaced by a biquad with transfer function

$$
b(s)=\frac{s \omega_{c}}{s^{2}+\omega_{0}^{2}} .
$$

A signal flow graph of a suitable biquad is depicted in Fig. 4. In this way, a bandpass filter is a network of biquads. The design of the biquads and the filter is discussed in the next section.

\section{THE FILTER}

The biquads of Fig. 4 have been realized with two integrators of the type shown in Fig. 1, and one inverter. A circuit diagram of the biquads is shown in Fig. 5. Two damping resistors of $200 \Omega$ are connected in series with the outputs to introduce high-frequency zeros that cancel parasitic poles. $T_{5}$ and $T_{9}$ of the inverter have been biased at a current that is 3.7 times higher than the bias current of the integrators. This has been done to reduce noise generation. Due to the noise production of the inverter, the effective noise factor of the integrator increases to 6.6.

The biquads must be mutually coupled, according to the signal flow graph of Fig. 3. For that purpose, an input and an output must be added to, or found in, the biquad.

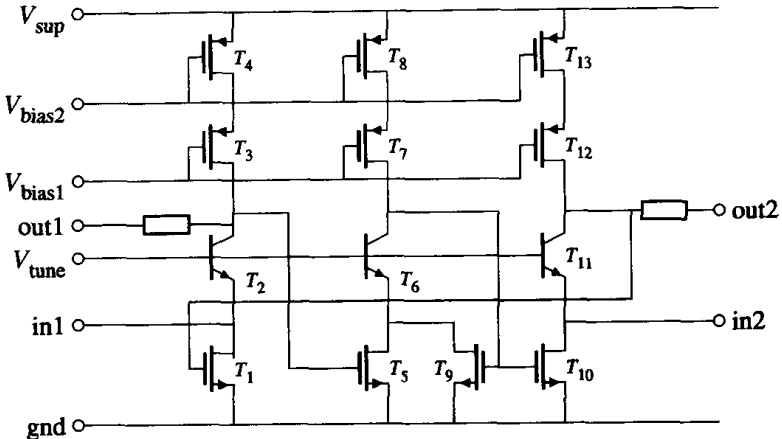

Fig. 5. The biquads consist of two transconductances of the type shown in Fig. 1 and an inverter. The capacitances are not shown in this figure, and must be connected to the outputs.

From a theoretical point of view, the most logical way of adding inputs to the biquad is by adding transistors in parallel to $T_{1}, T_{5}$, or $T_{10}$. The gates of these transistors can be used as extra inputs. The collectors of $T_{2}, T_{6}$, and $T_{11}$ can serve as outputs.

In practice, however, this scheme does not work. The transistors that must be added should be very long and narrow in order to realize the small transconductance values that are involved. Therefore, these transistors would add parasitic poles to the circuit. A simulation shows that these poles are so dominant that the transfer function cannot be realized in this way.

Therefore another method has been used. The inputs that are indicated in Fig. 5 are current inputs. If via a small series capacitance $C_{a_{i j}}$ an input voltage $V_{i n}$ is applied to input 1 in Fig. 5, as shown in Fig. 6, a current will enter this input. This current is proportional to the first derivative with respect to time of $V_{\text {in } 1}$. This current is passed through $T_{2}$, and integrated by the capacitor $C_{i}$ that is connected to output 1 . This voltage is inverted by the stage around $T_{5}$ and $T_{6}$, and integrated by the integrator around $T_{10}$. Thus the transfer function between input voltage $V_{\text {in } 1}$ and the voltage $V_{\text {out2 }}$ at output 2 is

$$
\frac{V_{\text {out2 }}}{V_{\text {in1 }}}=\frac{s \omega_{0} \frac{C_{a_{i j}}}{C_{i}}}{s^{2}+\omega_{0}^{2}}
$$

This is a noninverting transfer function. If input 2 and output 1 are used instead of the other two, an inverting transfer function is obtained:

$$
\frac{V_{\text {out } 1}}{V_{\text {in2 }}}=\frac{-s \omega_{0} \frac{C_{a_{i k}}}{C_{i}}}{s^{2}+\omega_{0}^{2}} .
$$

More inputs can be obtained by connecting more capacitances to the two inputs.

If in the filter network of Fig. 3 the integrators are substituted by biquads with transfer functions $b(s)$ as in (9), a network of multiple-input biquads with transfer func- 


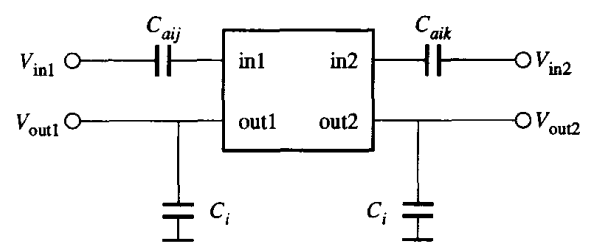

Fig. 6. The circuit of Fig. 5 used as a two-input two-output biquad.

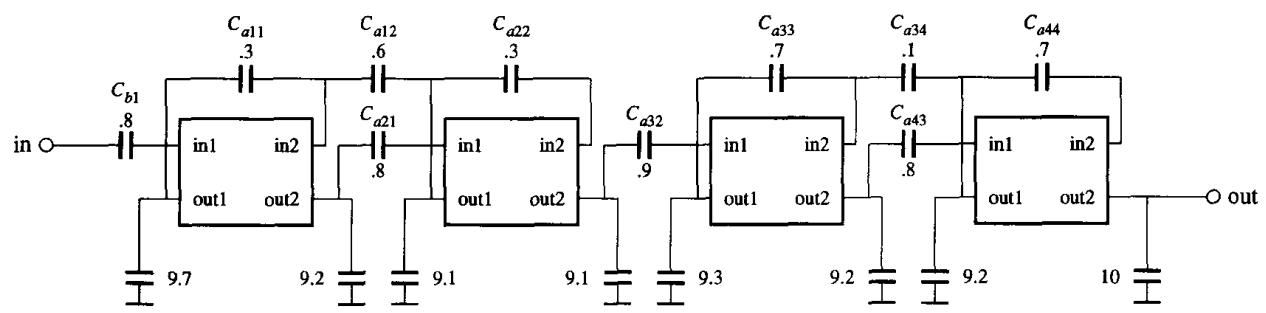

Fig. 7. The filter is a network of biquads of the type shown in Fig. 5. The capacitance values are in picofarads.

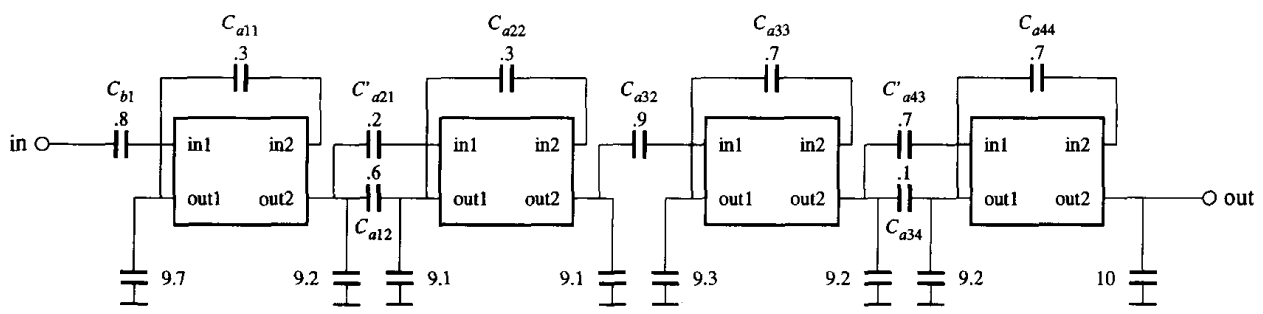

Fig. 8. The filter network of Fig. 7 modified to further reduce the influence of parasitics.

tions $a_{i j}^{\prime \prime} b(s)$ is obtained. This, with (10) and (11), yields

$$
C_{a_{i j}}=\frac{C_{i}\left|a_{i j}^{\prime \prime}\right|}{Q}
$$

in the filter realization of Fig. 7.

In simulations it appears that the transfer function still is slightly affected by parasitic poles in the couplings between the biquads. The influence of these parasitics can be further reduced by modifying the circuit of Fig. 7. It can be proved that two cross-coupled capacitors $C_{a_{i j}}$ and $C_{a_{j i}}$ can be replaced by one capacitor $C_{a_{i j}}$ directly connected between two outputs of the two biquads involved, if these two capacitors have the same value. If the values of the two capacitors are unequal, the smallest of them can be connected between the outputs, if this value is subtracted from the largest coupling capacitor. This results in the filter of Fig. 8. When this network is simulated, it appears that the effects of parasitics has been reduced to an acceptable degree.

The capacitors were dimensioned such that at each output of each biquad a total capacitance of $10 \mathrm{pF}$ was situated. A high degree of matching between the biquads is necessary. Therefore, the loading capacitances (of $10 \mathrm{pF}$ ) of each biquad were realized as a parallel connection of one 9.1-pF sandwich capacitor with nine $0.1-\mathrm{pF}$ top-plate capacitors. The small capacitors could be earthed or connected to the input or output of another stage for coupling if wanted. In this way the total capacitance values are well matched.

The bias voltages required by the circuit in Fig. 5 are generated by the circuit in Fig. 2 . The central frequency of the filter can be tuned with constant $Q$ via $V_{\text {tune }}$. The bias lines of all the biquads were tied together and connected to one bias generating circuit. In this situation there is some crosstalk over the bias lines. It appeared in simulations and measurements that this does not degrade the stopband damping very much. This crosstalk also has an influence on the passband shape of the filter, because it affects the intercoupling of the biquads. This influence also appeared to be small and acceptable. In situations where the crosstalk via the bias lines has too much effect, it should be eliminated. This can be done by furnishing each biquad with a separate bias generator.

A chip photograph of the filter is shown in Fig. 9. On this chip the eighth-order filter, together with a secondorder reference filter, are realized, but on the photograph only the eighth-order filter is shown. The chip area occupied by this filter is only $0.25 \mathrm{~mm}^{2}$. Near the bottom of the picture, the $10-\mathrm{pF}$ capacitors that each consist of a large capacitor together with nine small capacitors are clearly visible. 


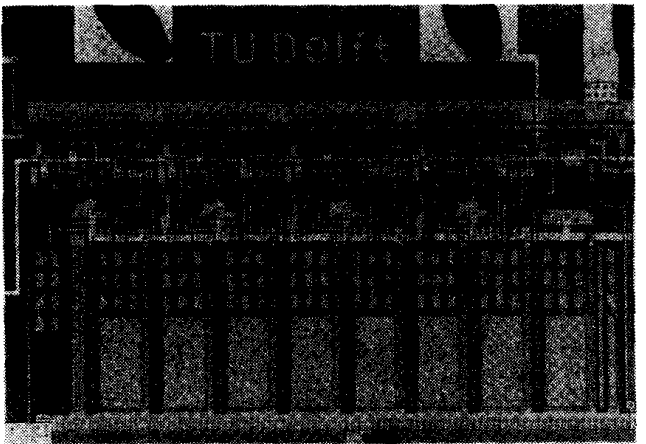

Fig. 9. The filter chip.

\section{Measurements}

The supply current of the chip (the eighth-order filter together with the second-order reference filter) is 1.00 $\mathrm{mA}$. As the supply voltage is $8.0 \mathrm{~V}$ the power consumption is $8.0 \mathrm{~mW}$.

Fig. 10 shows the measured transfer function of the filter, accurately representing the design objective. There is a slight tilt of $0.2 \mathrm{~dB}$ visible in the transfer function, and if necessary this tilt can be removed by giving each biquad its own bias generator, as was explained in Section IV. The stopband damping is $70 \mathrm{~dB}$.

The output noise voltage was measured to be $708 \mu \mathrm{V}$. Theoretically, this should be $553 \mu \mathrm{V}$ [4]. The difference is ascribed to the fact that the constant $c$ in (3) is 1.1 instead of $2 / 3$.

The maximal effective output signal voltage is $0.9 \mathrm{~V}$ by measurements. This is less than the $1-\mathrm{V}$ maximal signal level of one single integrator. The difference is ascribed to a mismatch in bias voltages between the integrators. The dynamic range is therefore $62 \mathrm{~dB}$, which is $3 \mathrm{~dB}$ less than predicted in Section III. The distortion-free dynamic range is $47 \mathrm{~dB}$. This was measured on the basis of thirdorder intermodulation distortion because, in a high- $Q$ bandpass filter, this is the most important distortion mechanism. The tuning range is $50-200 \mathrm{kHz}$.

\section{Improving The Dynamic Range}

There are several ways to improve the dynamic range of the filter that we have designed. In this section we will discuss them briefly and give a fundamental maximum for the dynamic range. From (7) and (8) one sees that the dynamic range increases if the total amount of capacitance or the maximal signal voltage is increased, or if the quality factor of the filter is decreased. The maximal signal voltage depends in most cases on the supply voltage, so the maximal signal voltage can be enlarged if the supply voltage is enlarged. Because these methods to improve the dynamic range are trivial, we will assume that the total amount of capacitance, the supply voltage, and the transfer function of the filter cannot be changed.

In this section we deal with dynamic range only. As a rule of the thumb, each improvement of $3 \mathrm{~dB}$ in dynamic

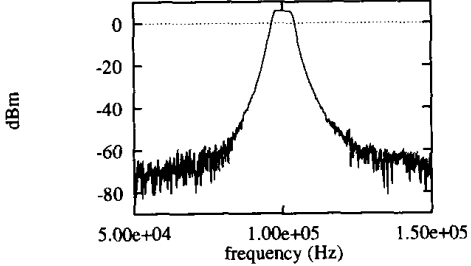

(a)

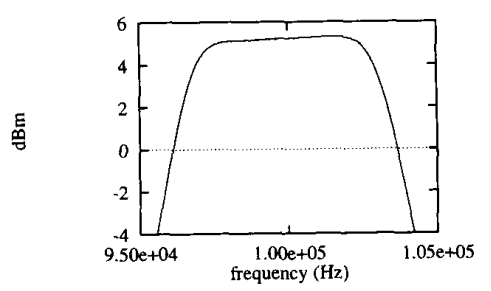

(b)

Fig. 10. The transfer function of the filter by measurements. In (a) the transfer function in a wide frequency range is shown, while (b) shows the passband in more detail.

range gives rise to an improvement of $2 \mathrm{~dB}$ in third-order distortion-free dynamic range, but this is not always true. A more fundamental way of improving the distortion-free dynamic range is improving the linearity of the integrators, for which device modeling needs to be done [22].

One way to improve the dynamic range is network optimization. It was pointed out in Section III that a maximum of $0.6 \mathrm{~dB}$ can be gained in this way.

More improvement can be obtained if the integrators are optimized. The amount of dynamic range that can be won this way depends upon which degrees of freedom one has. If one has the possibility of designing the MOSFET's for this goal, better results can be obtained than with existing MOSFET's, as was our situation. Theory on optimal dynamic range integrators has been described elsewhere [3]. Here the main results for this practical situation are summarized.

An improvement of $6 \mathrm{~dB}$ can be obtained by making the integrator differential, at the cost of more circuit complexity. This is shown in Fig. 11. The improvement is due to an increase of the maximal signal levels by $6 \mathrm{~dB}$.

The noise factor can be reduced by effectively eliminating the noise production by the bias current sources in the integrator. The integrator of Fig. 1 contains one bias current source that is realized with the MOSFET's $T_{3}$ and $T_{4}$, and that produces noise. This gives rise to an increase in the noise factor, an increase that can be circumvented by using a complementary circuit as shown in Fig. 12. A similar thing has been done by Nauta [10], [25], Nauta and Seevinck [11], and Park and Schaumann [19] for saturation-region transconductors. These saturation-region transconductors have better high-frequency potentials [10], whereas this triode-region version can have a larger dynamic range [3], [4].

If the supply voltage is $8 \mathrm{~V}$, an optimal value for the threshold voltage is about $-0.17 \mathrm{~V}$, with an optimal value 


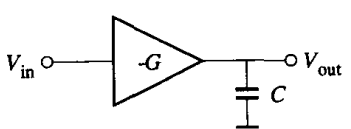

(a)

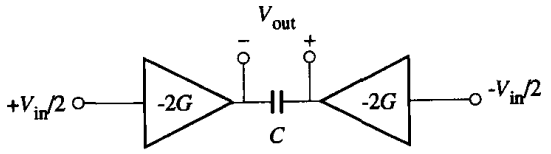

(b)

Fig. 11. A dynamic-range improvement can be obtained if the integrators are made differential (b) instead of single ended (a).

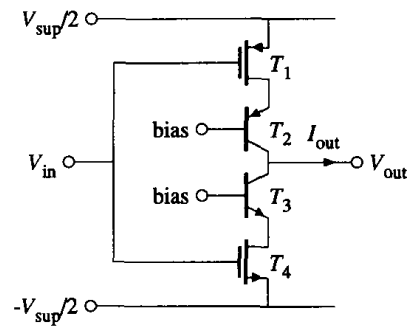

Fig. 12. A noise-producing bias current source in the integrator can be circumvented by using a complementary transadmittance stage.

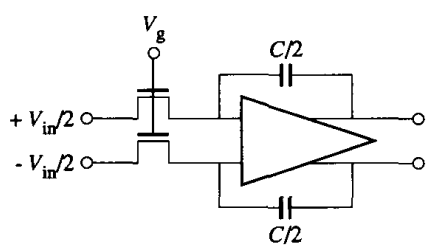

Fig. 13. A MOSFET-C integrator.

for the drain-source voltage of $1.5 \mathrm{~V}$ [3]. The noise factor is then 2.35 and, if the stage is used in a differential mode, the maximal differential signal amplitude is $5.1 \mathrm{~V}$; assuming sinusoidal signals, this means a maximal effective signal voltage of $3.6 \mathrm{~V}$. With a capacitance of $10 \mathrm{pF}$, this gives rise to a dynamic range of $103 \mathrm{~dB}$ for the integrator, an increase of $14 \mathrm{~dB}$ when compared to the integrator we have used, but complementary transistors and MOSFET's with a suitable threshold voltage must be available. By (7), the dynamic range of the filter would become $81 \mathrm{~dB}$.

There is an upper limit to the dynamic range, and this limit can be reached in several ways. One way is by use of a MOSFET-C integrator with optimized MOSFET's. A MOSFET-C integrator is shown in Fig. 13. If the differential op amp in this integrator can drive its output rail to rail, and the threshold voltage of the MOSFET's is -8 $\mathrm{V}$, the maximal single-ended input and output amplitude of the integrator is equal to half the supply voltage. The noise factor can be made equal to 1 , and this gives, according to (7), rise to a dynamic range of $82.4 \mathrm{~dB}$ for the filter. If additionally an optimal filter network is used, the dynamic range will be $83.0 \mathrm{~dB}$, according to (8). This is the largest dynamic range that can ever be reached for an active eighth-order Butterworth bandpass filter with a quality factor of 14.3 , a total capacitance of $80 \mathrm{pF}$, and a supply voltage of $8 \mathrm{~V}$.

\section{Conclusions}

With very simple circuitry it is possible to realize an eighth-order high- $Q$ bandpass filter with a large tuning range, a high dynamic range, and a very small chip area. The dynamic range can be improved by $21 \mathrm{~dB}$ to a theoretical maximum of $83 \mathrm{~dB}$, mainly by redesigning the integrators, at the cost of a more complex circuit. If still a larger dynamic range is wanted, this can only be attained by increasing the supply voltage or by enlarging the total capacitance. The latter must be paid in chip area and power consumption.

\section{ACKNOWLEDGMENT}

The chip has been processed by Philips, for which the author wishes to express his gratitude.

\section{REFERENCES}

[1] Y. Tsividis, M. Banu, and J. Khoury, "Continuous-time MOSFETC filters in VLSI," IEEE Trans. Circuits Syst., vol. CAS-33, pp. 125-140, Feb. 1986.

[2] G. Groenewold, "The design of high dynamic range continuous-time integratable bandpass filters," IEEE Trans. Circuits Syst., vol. 38 , pp. 838-852, Aug. 1991.

[3] G. Groenewold, "Optimal dynamic range integrators,"' submitted to IEEE Trans. Circuits Syst.

[4] G. Groenewold, "Optimal dynamic range integrated continuous-time filters," Ph.D. dissertation, Delft Univ. Technology, Delft, The Netherlands, 1992.

[5] K.-S. Tan and P. R. Gray, "Fully integrated analog filters using bipolar-JFET technology," IEEE J. Solid-State Circuits, vol. SC-13, no. 6 , pp. 814-821, Dec. 1978

[6] M. Banu and $Y$. Tsividis, "Fully integrated active $R C$ filters in MOS technology," IEEE J. Solid-State Circuits, vol. SC-18, no. 6, pp. 644-651, Dec. 1983.

[7] M. Banu and Y. Tsividis, "An elliptic continuous-time CMOS filter with on-chip automatic tuning," IEEE J. Solid-State Circuits, vol. SC-20, no. 6, pp. 1114-1121, Dec. 1985.

[8] F. Krummenacher and N. Joehl, "A 4-MHz CMOS continuous-time filter with on-chip automatic tuning," IEEE J. Solid-State Circuits, vol. 23 , pp. $750-758$, June 1988 .

[9] A. Kaiser, "A micropower CMOS continuous-time low-pass filter," IEEE J. Solid-State Circuits, vol. 24, no. 3, pp. 736-743, June 1989.

[10] B. Nauta, "CMOS VHF transconductance-C lowpass filter," Electron. Lett., vol. 26, no. 7, pp. 421-422, Mar. 1990.

[11] B. Nauta and E. Seevinck, "A 110MHz CMOS transconductance-C lowpass filter," in Conf. Proc. ESSCIRC (Vienna), 1989, pp. 141144

[12] V. Gopinathan, Y. P. Tsividis, K.-S. Tan, and R. K. Hester, "Design considerations for high-frequency continuous-time filters and implementation of an antialiasing filter for digital video," IEEE J. SolidState Circuits, vol. 25, no. 6, pp. 1368-1378, Dec. 1990.

[13] J. van der Plas, "MOSFET-C filter with low excess noise and accurate automatic tuning," IEEE J. Solid-State Circuits, vol. 26, pp. 922-929, July 1991.

[14] A. van Bezooijen, N. Ramalho, and J. O. Voorman, "Balanced integrator filters at video frequencies," in Conf. Proc. ESSCIRC (Milan), 1991, pp. 1 4. 
[15] M. Steyaert, J. Silva-Martinez, and W. Sansen, "High-performance OTA-RC continuous-time filters with full CMOS low distortion floating resistors," in Conf. Proc. ESSCIRC (Milan), 1991, pp. 5-8.

[16] R. Alini, A. Baschirotto, and R. Castello, " $8-32 \mathrm{MHz}$ tunable BiCMOS continuous-time filter," in Conf. Proc ESSCIRC (Milan), 1991, pp. 9-12.

[17] G. J. Smolka, U. Grehl, B. Jahn, U. Riedle, W. Veit, and H. Werker "A low-noise trunk interface circuit," in Conf. Proc. ESSCIRC (Milan), 1991, pp. 257-260.

[18] H. Khorramabadi and P. R. Gray, "High-frequency CMOS continuous-time filters,' IEEE J. Solid-State Circuits, vol. SC-19, no. 6 pp. 939-948, Dec. 1984.

[19] C. S. Park and R. Schaumann, "Design of a 4-MHz analog integrated CMOS transconductance-C bandpass filter," IEEE J. Solid-State Circuits, vol. 23, pp. 987-996, Aug. 1988.

[20] T. G. Kim and R. L. Geiger, "Monolithic programmable RF filter," Electron. Lett., vol. 24, no. 25, pp. 1569-1571, Dec. 1988.

[21] Y. P. Tsividis, Operation and Modeling of the MOS Transistor. New York: McGraw-Hill, 1987.

[22] G. Groenewold and W. J. Lubbers, "Designing low distortion analogue integrators with use of a new MOSFET model,"' submitted to IEEE Trans. Circuits Syst.
[23] J. L. Pennock, "CMOS triode transconductor for continuous-time active integrated filters," Electron. Lett., vol. 21, no. 18, pp. 817-818, Aug. 1985.

[24] S. L. Wong, "Novel drain-biased transconductance building blocks for continuous-time filter applications," Electron. Lett., vol. 25, no. 2, pp. 100-101, Jan. 1989.

[25] B. Nauta, "A CMOS transconductance-C filter technique for very high frequencies," IEEE J. Solid-State Circuits, vol. 27, pp. 142153, Feb. 1992

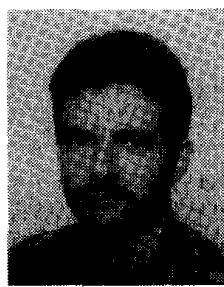

Gert Groenewold received the M.S. degree in electrical engineering from the Delft University of Technology in Delft, The Netherlands, in 1988. Subsequently he joined the Electronics Laboratory of the same university where he received the $\mathrm{Ph} . \mathrm{D}$. degree in 1992. His research includes developing a formal design theory for analog integrated filters along with the development of software. 